\title{
HYDROSTATIC PRESSURE STUDIES OF ASYMMETRIC DOUBLE-BARRIER RESONANT TUNNELING DIODES*
}

R. Diniz, J. SMoliner, E. Gornik

Walter Schottky Institut, TU München, 8046 Garching, Am Coulombwall, Germany T. SUSKI

UNIPRESS, Polish Academy of Sciences, Sokolowska 29/37, 01-142 Warszawa, Poland

\section{U. Meiners and H. Brugger}

Daimler-Benz Research Centre, 7900 Ulm, PO Box 2360, Germany

Tunneling processes in double-barrier GaAs/AlAs diodes with an incorporated $A l G a A s$ pre-barrier were studied under hydrostatic pressure. The electrical characteristics resulting from a pre-barrier on the side of the emitter can be explained at 1 bar, solely by the $\Gamma$-profile: increasing pressure shows that the pre-barrier does not reduce the $\Gamma$-X tunneling. A pre-barrier on the collector side leads to charge buildup at the $X$ minimum within the AlAs collector barrier.

PACS numbers: 72.80.Ey, 73.40.Gk

The physical properties of double-barrier resonant tunneling diodes (DBRTDs) have been extensively studied [1,2] and considered for device applicaiions [3, 4]. A DBRTD is formed by a low-gap semiconductor material (e.g. GaAs) between two layers of a higher-gap one (e.g. AlAs). Its $\Gamma$ conduction-band profile consists of two barriers sandwiching a quantum well whose energy levels manifest themselves as resonance peaks in the current density-voltage $J(V)$ characteristics. However, since AlAs is an indirect gap semiconductor, additional tunneling through the $X$ valleys in the barriers can influence the whole transport mechanism [5-9]. This paper presents data on the effect of hydrostatic pressure $(p)$ on a GaAs/AlAs DBRTD with an AlGaAs additional layer. This kind of structure was reported to improve both peak current and peak to valley ratio (PVR), which are key parameters for device performance $[10,11]$.

Sample $A$ consists of a $17 \AA / 50 \AA / 17 \AA$ AlAs/GaAs/AlAs DBRTD with an $\mathrm{Al}_{0.2} \mathrm{Ga}_{0.8} \mathrm{As}$ pre-barrier layer (see inset in Fig. 1a) [11]. Sample $B$ is a reference without pre-barrier. At $p=1$ bar and zero external bias, the $X$ and $\Gamma$ minima

*This work was partially supported by CNPq (Brazil), Committee for Scientific Research (Poland) and Verbund Vorhaben III-V Elektronik Mesoskopische Bauelemente (Germany). 


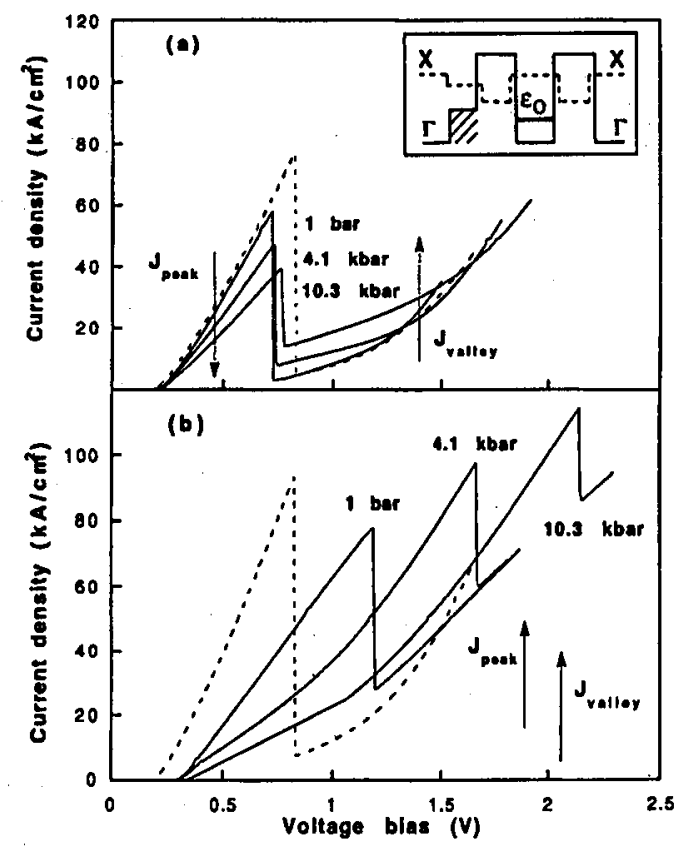

Fig. 1. (a) Current density versus voltage $J(V)$ curves at $T=4.2 \mathrm{~K}$ for pressures equal to $p=1 \mathrm{bar}, 4.1 \mathrm{kbar}$, and $10.3 \mathrm{kbar}$ for the DBRTD with pre-barrier in emitter $(A+$, forward bias). The peak current density decreases and the valley current density increases with pressure (see arrows). The insert shows the schematic band edge profile ( $\Gamma$ and $X$ points correspond respectively to solid and broken lines) of a DBRTD with a pre-barrier (hatched region). (b) Current density versus voltage $J(V)$ curves at $T=4.2 \mathrm{~K}$ in absolute value for pressures equal to $p=1 \mathrm{bar}, 4.1 \mathrm{kbar}$, and $10.3 \mathrm{kbar}$ for the DBRTD with pre-barrier in collector ( $A-$, backward bias). Both current densities increase with pressure (see arrows). The broken lines represent in (a) and (b) $J(V)$ for sample $B$ at $p=1$ bar and $T=4.2 \mathrm{~K}$.

in the AlAs barriers lie respectively about $160 \mathrm{meV}$, and $1.05 \mathrm{eV}$ above the $\Gamma$ minimum in GaAs [5]. Since the same minima lie at 362 and $140 \mathrm{meV}$ respectively in AlGaAs, the pre-barrier builds a chair in both $\Gamma$-and $X$-profiles. The first resonant energy level lies at $\varepsilon_{0} \approx 105 \mathrm{meV}$.

Figure la shows the $J(V)$ curves of sample $A$ with the pre-barrier in emitter (forward bias, $A+$ ) and collector side (reverse bias, $A-$ ) for three different pressures at $T=4.2 \mathrm{~K}$. A comparison of $A+$ with $B$ shows that the effect of the pre-barrier at forward bias and $p=1 \mathrm{bar}$ is to reduce peak ( $\left.J_{\text {peak }}\right)$ and valley current densities ( $J_{\text {valley }}$ ). The pre-barrier in emitter improves the PVR at $T=4.2 \mathrm{~K}$ from $12(B)$ to $18(A+)$. Similar results have been attributed to a reduction of both coherent tunneling and tunneling through the $X$ valleys [10]. Sample $B$ exhibits a very symmetric behavior with respect to the direction of applied bias voltage. The roles played by $\Gamma-\Gamma$ and $\Gamma-X$ tunneling can be better determined by increasing 
$p$. For $A+$ at forward bias, $J_{\text {peak }}$ decreases and $J_{\text {valley }}$ increases with $p$, respectively. IIydrostatic pressure reduces the $\Gamma-X$ offset at a rate of $\mathrm{d}\left(\varepsilon_{X}-\varepsilon_{\Gamma}\right) / \mathrm{d} p \approx$ $-11 \mathrm{meV} \mathrm{kbar}^{-1}$ and about maintains the $\Gamma$-band discontinuity. An increase in $p$ is also followed by an increase in the $\Gamma$ effective masses. Since $J_{\text {peak }}$ is mainly determined by $\Gamma-\Gamma$ tunneling, the increase in effective mass explains the observed decrease in $J_{\text {peak }}$ with $p[1,5] . J_{\text {valley }}$ is determined mainly by indirect tunneling through the $X$ point and its increase with $p$ results from the decrease in the height of the $\Gamma-X$ barriers [5-9]. If $J_{\text {peak }}$ and $J_{\text {valley }}$ are normalized by their values at $p=1$ bar, a comparison between both samples (not shown here) indicates that $J_{\text {peak }}$ decreases with the same pressure rate for $A+$ and $B$. On the other hand, $J_{\text {valley }}$ increases more rapidly for $A+$ than for $B$. Both results do not confirm the reduction of $\Gamma-X$ tunneling after the addition of the pre-barrier layer. In fact, the observed decrease in $J_{\text {peak }}$ at $p=1$ bar for $A+$ in comparison to $B$ is due to the increase in the $\Gamma$ emitter barrier thickness. An increase in barrier thickness also leads to a decrease in the resonant energy level mean width $\left(\Delta \varepsilon_{0}\right)$. As PVR $\propto \Delta \varepsilon_{0}^{-1}$, the increase in PVR from $B$ to $A+$ can be explained solely by the $\Gamma$ profile. This point is also confirmed by analysis of the dependence of the PVR on $p$ : although $A+, A-$ and $B$ have very different PVRs at $p=1$ bar, resonance disappears at the same critical pressure of $p \approx 14.3 \mathrm{kbar}$ for all configurations. This again can be taken as an evidence that the $X$ contribution to tunneling is similar for both samples.

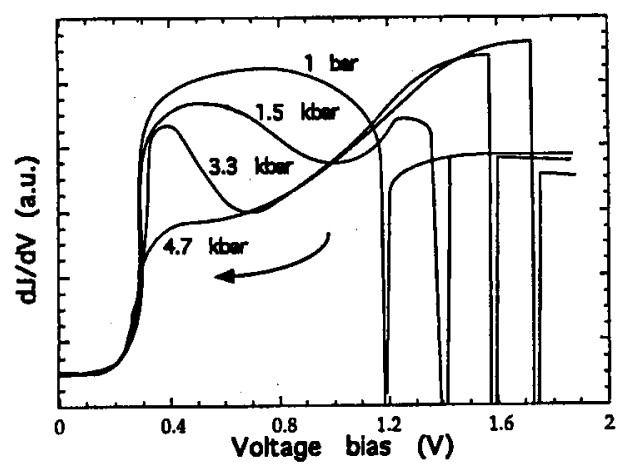

Fig. 2. Conductivity versus voltage $\mathrm{d} J / \mathrm{d} V$ curves (in arbitrary units) for $A-$ as a function of pressure for $p=1 \mathrm{bar}, 1.5 \mathrm{kbar}, 3.3 \mathrm{kbar}$, and $4.7 \mathrm{kbar}$ at $T=4.2 \mathrm{~K}$. The arrow represents the change in position of the $\mathrm{d} J / \mathrm{d} V$ minimum with increasing pressure.

Peak voltage $\left(V_{\text {peak }}\right)$ as well as $J_{\text {peak }}$ and $J_{\text {valley }}$ increase with $p$ when the pre-barrier is on the collector side (Fig. $1 \mathrm{~b}$, reverse bias). The usual triangular shape of the $J(V)$ curves at $p=1$ bar change into a concave shape with increasing $p$. Figure 2 shows the conductivity $\mathrm{d} J(V) / \mathrm{d} V$ curves for $A-$ at $T=4.2 \mathrm{~K}$ as a function of $p$. A knee appears before resonance and it turns into a local minimum with increasing pressure. This minimum delimitates two regions at the conductivity curves and its bias position decreases with $p$ until it disappears for $p>4.7 \mathrm{kbar}$. 
Note that the $X$ point at the AlAs barriers has, for $p \geq 2 \mathrm{kbar}$, already crossed the $\Gamma$ point at the pre-barrier. Beyond this pressure, the Fermi energy in the emitter must cross the $X$ point in the AlAs collector barrier before resonance. Within the picture of sequential tunneling, the AlGaAs layer acts as an additional barrier in the collector for electrons tunneling through the $X$ valley, and charge buildup at the $X$ point in the collector AlAs barrier is originated. Similarly to buildup in the central well [2], the presence of charges within this barrier decreases the electric field at the emitter region and increases it at the collector. Higher bias will be needed for resonance and the second barrier also becomes more "transparent". As shown by Fig. 1 for backward bias, increased pressures lead for $A$ - to an increase in $V_{\text {peak }}$ and $J_{\text {peak }}$. The position of the $X$ point at the barriers decreases with $p$ until it crosses the quasi-bound $\varepsilon_{0}$ level at $p \approx 5.0$ kbar. As shown by Fig. 2, above this pressure charge buildup occurs at very low bias already, and the minimum at the $\mathrm{d} J / \mathrm{d} V$ curves can no longer be observed. As a summary, hydrostatic pressure applied to a DBRTD with an AlGaAs pre-barrier in the emitter does not show reduction of indirect $\Gamma-X$ tunneling. Electrical characteristics of the sample can be explained in this case solely by the $\Gamma$ potential profile. The presence of the pre-barrier on the collector side originates charge buildup on the $X$ point at the AlAs barriers. This phenomenon becomes more pronounced by increasing the external pressure.

\section{References}

[1] E.E. Mendez, Physics and Applications of Quantum Wells and Superlattices, Eds. E.E. Mendez, K. von Klitzing, Plenum Press, New York 1987, p. 159.

[2] G.A. Toombs, F.W. Sheard, Electronic Properties of Multilayers and Low-Dimensional Structures, Eds. J.M. Chamberlain et al., Plenum Press, New York 1990, p. 257.

[3] F. Capasso, K. Mohammed, A.Y. Cho, IEEE J. Quantum Electron. 22, 1853 (1986).

[4] T.C.L.G. Sollner, E.R. Brown, C.D. Parker, W.D. Goodhue, in Ref. [2], p. 283.

[5] E.E. Mendez, E. Calleja, W.I. Wang, Phys. Rev. B 34, 6026 (1986).

[6] D.G. Austing, P.C. Klipstein, A.W. Higgs, G.W. Smith, J.S. Roberts, G. Hill, Resonant Tunneling in Semiconductors - Physics and Applications, Eds. L.L. Chang et al., Plenum Press, New York 1991, p. 157.

[7] L.A. Cury, L. Dmowski, A. Celeste, J.C. Portal, D.L. Svico, A.Y. Cho, T.J. Foster, L. Eaves, M. Davies, M. Heath, J.R. Middleton, Semicond. Sci. Technol. 6, 499 (1991).

[8] R. Diniz, T. Suski, K. Berthold, J. Smoliner, E. Gornik, H. Riechert, Semicond. Sci. Technol. 7, 271 (1992).

[9] R. Diniz, J. Smoliner, E. Gornik, U. Meiners, H. Brugger, T. Suski, P. Wisniewski, to be published in Semicond. Sci. Technol. 8, (1993).

[10] P. Cheng, J.S. Harris, Jr., Appl. Phys. Lett. 56, 1676 (1990).

[11] H. Brugger, U. Meiners, C. Wölk, R. Deufel, J. Schroth, A. Förster, H. Lüth, in: Proc. 13th IEEE/Cornell Conf. on Adv. Concepts in High Speed Semicond. Dev. and Circ., 1991, p. 39.

[12] S. Adachi, J. Appl. Phys. 58, R1 (1985). 